\title{
Evaluating Visitor Experiences with Interactive Art
}

\author{
Fabio Morreale \\ interAction research group \\ University of Trento \\ Italy \\ morreale@disi.unitn.it
}

\author{
Antonella De Angeli \\ interAction research group \\ University of Trento \\ Italy \\ deangeli@disi.unitn.it
}

\begin{abstract}
The Music Room is an interactive installation that allows visitor to compose classical music by moving throughout a space. The distance between them and their average speed maps the emotionality of music: in particular, distance influences the pleasantness of the music, while speed influences its intensity. This paper focuses on the evaluation of visitors' experience with The Music Room by examining log-data, video footages, interviews, and questionnaires, as collected in two public exhibitions of the installation. We examined this data to the identify the factors that fostered the engagement and to understand how players appropriated the original design idea. Reconsidering our design assumptions against behavioural data, we noticed a number of unexpected behaviours, which induced us to make some considerations on design and evaluation of interactive art.
\end{abstract}

\section{CCS Concepts}

- Human-centered computing Field studies - Applied computing Performing arts - Applied computing $\sim$ Sound and music computing • Applied computing $\sim$ Media arts

\section{Keywords}

Evaluation methods, interactive installations, audience engagement

\section{INTRODUCTION}

In recent years, the HCI community has been embracing works born from the combination of interaction design and art $[8,9,19]$. The peculiar aspect of these works lies in the active role of the user in the creation of the actual artefacts [18]. In most cases, the artefact is composed of audio-visual material [11] and, at times, it specifically focuses on allowing users to actively interact with musical contents [4]. Following this research direction, we developed The Music Room, an interactive installation that makes musical expression accessible even to musically untrained users $[21,22]$. By moving in pair inside a given space, visitors can direct the music, which is co-created with an algorithmic composer.

After the the installation was exhibited during two public events, much effort was put into the evaluation of visitor experiences. Understanding user experience is indeed critical in all HCI branches [12], even more so when it comes to interactive art, where the meaning and the scope of the artwork are explicitly co-created

Permission to make digital or hard copies of all or part of this work for personal or classroom use is granted without fee provided that copies are not made or distributed for profit or commercial advantage and that copies bear this notice and the full citation on the first page. Copyrights for components of this work owned by others than ACM must be honored. Abstracting with credit is permitted. To copy otherwise, or republish, to post on servers or to redistribute to lists, requires prior specific permission and/or a fee. Request permissions from Permissions@acm.org.

CHItaly 2015, September 28-30, 2015, Rome, Italy

(C) 2015 ACM. ISBN 978-1-4503-3684-0/15/09 ..\$15.00

DOI: $\underline{\text { ttp://dx.doi.org/10.1145/2808435.2808440 }}$ with visitors [1]. Accordingly, accurate tests on the system quality must be complemented by a thorough investigation of the visitor behaviour in order to effectively frame the status of the work itself. Yet, most of the related work paid little attention to the latter aspect.

This paper suggests a number of methods to understand user experience in interactive art. Two field evaluations were conducted during two live events open to the general public $(\mathrm{N}=344)$. An integration of online observations, interviews, questionnaires, and offline analysis of $\log$ data and videos was performed. Results suggested that nearly all visitors experienced authentic enjoyment. Musically untrained visitors, in particular, referred to the experience as remarkably creative. The analysis also offered insights into the behaviours of visitors, who engaged with the installation in a number of different ways, at times appropriating the original design idea.

This paper pursues a twofold aim: on the one hand, it offers a holistic evaluation approach to the cost-benefit analysis of different data gathering techniques, which readers interested in the design of artistic and interactive installations may benefit from. Specifically, we aimed to explain the success of The Music Room by integrating evidences collected through an array of evaluation techniques borrowed from the field of user-experience [7, 12]. On the other hand, it provides follow-up discussions about the consequences of open-ended design in the context of interactive art. Unbiased by any pre-conceived interpretations, our visitors were free to gain their own understanding of the system.

The paper is organised as follows. Section 2 reviews the theoretical foundation of this work and analyses the current state of research in musical interface design. Section 3 presents the conceptual design and the technical implementation of The Music Room. Section 4 features an evaluation of the experience of visitors, suggesting new insights into the way people experience interactive art. Section 5 investigates the implications of this work for the research areas of interactive art.

\section{RELATED WORK}

Interest in the design of interfaces for music making has been growing in the last decade [2]. The related work can be clustered into two categories [23]: i) digital musical interfaces, and ii) interactive musical installations. The design of the Music Room was partially inspired by works conducted in both fields.

The first category counts a number of new virtual instruments and controllers, whose user interaction resembles that of traditional instruments [32]. In the Reactable, for example, the music is directed by controlling tangible blocks that are associated with specific acoustic features or musical parameters [15]. Similarly, the AudioPad allows performers to control sound synthesis via tangible interaction [25]. Unlike traditional instruments, these systems encode users' musical meanings into high-level musical structures (e.g. sequencers, scale selection, envelopes, loops) rather than operating on low-level musical parameters. As a consequence, the cognitive and motor efforts required for making music are 
decreased. High-level musical structures, though, are still unlikely to convey any meaning to musically untrained users.

The second category counts a handful of interactive systems that specifically address naïve listeners. Several of them exploit the concept of active listening [27]: users can interactively control the musical content by modifying it in real time, while they are listening to it [4]. On the basis of this concept, several works sought to enable people to shape musical contents through collaborative interaction [5]. Mappe per Affetti Erranti, for example, allows people to explore pre-composed music by moving in the physical space [5]. Collaboration among the users is highly encouraged, as they can only appreciate music in its full complexity if they cooperate with each other by moving throughout the space. This collaborative approach was also adopted in TouchMeDare [30]. Two or more people can make music by interacting through a canvas, and pre-composed music samples are only triggered when the canvas is simultaneously touched by more than one user.

The interaction metaphors proposed by these works have contributed in different ways to empower musically untrained users to actively operate on musical elements. However, in most studies, the design process of the interfaces is only partially described, hampering the establishment of best practices and failing to provide useful insights for other researchers interested in building similar interfaces. Moreover, there is very little if any evaluation of the player's experience. In some exceptional cases, the evaluation is limited to administering questionnaires $[30,31]$ or to testing the quality of the system [10]. To tackle the lack of user experience evaluation in related musical interfaces, the broader design space of interactive art was examined, as to to review methods for the user experience analysis.

Although the interest of the scientific community in interactive art is slowly but steadily growing [9], specific challenges related to understanding audience experience and engagement remain unsolved [8]. Over the last few years, designers and artists have come to recognise the need to evaluate experience with interactive installations [8] using a diversity of methods. [6], for instance, reviewed research methods most often adopted to study interactive artworks: direct observation, observation via video recording, contextual interviews, and video-cued recall. As an alternative, following an ethnography-inspired approach, [24] command the artist to become a hidden participant (shadowing), take part in informal discussions with participants in situ, and ask them to complete formal questionnaires.

A general consensus suggests that engagement with interactive art widely varies among people [25]. It would be inappropriate to expect user experiences to be uniform and to only consider an artwork successful if every participant has engaged with it as expected by the designer. Design should in fact anticipate multiple interpretations of the system [29] given by the "user's interpretation, understanding, attitudes, personality and expectations of computer culture" [13]. Elaborating on the role of subjective variability, [1] highlighted the importance of subverting expectations to foster engagement with interactive art. In their view, engagement is a transformative dialogue between the participant and the artwork. Participants explore installations based on their expectations: if expectations are not met, they may become curious, continue exploring the system and eventually reconsider their intentions and expectations. Accordingly, keeping the ultimate purpose of the installation deliberately ambiguous can foster creative engagement, which, in turn, is conducive to creative outcomes and/or increased understanding.
Combining suggestions from related work with original contributions, this paper infers from the case of the Music Room a number of methods to support the evaluation of the experience of visitors with interactive art. In particular, we propose the integration of evidence from quantitative and qualitative methods to understand audience experience and engagement.

\section{DESIGN}

The Music Room is an interactive installation designed to provide naïve visitors with a creative experience of music making. The installation was designed to be experienced by pairs of visitors, which can direct the emotional character of music by means of their movements in the room. In order to convey the desired emotions in an intuitive and engaging manner, we adopted the metaphor of intimacy. Their distance influences music mood: the more proximal the visitors are, the more positive the music. Their speed influences music intensity: the faster they move, the louder and faster the music.

The project, whose design details were analysed in a related article [22], was conceived from the idea of one of the authors and it iteratively evolved during two years of research through design, a practice intended to exploit design to produce knowledge [33]. The two years of project development started with a conceptual design stage. During this phase, three basic scenarios were envisaged: music making, dancing, and acting. In the music making scenario, visitors would enjoy trying all possible combinations of distance and speed to produce changes in the music. In the dancing scenario, visitors would spend most of the time dancing to the music they were composing. In the acting scenario, visitors would act out a drama while creating a soundtrack at the same time.

The scenarios were enriched with graphics and storyboards (Figure 1) and used as probes in a workshop involving 6 user experience researchers, 2 musicians, 2 designers and 2 interactive artists. The participants supported the conceptual idea of The Music Room, elaborating a number of interesting considerations. In particular, they were keen to discuss the possible behaviours of the people in the room - whether they would be more interested in creating music or enjoying the intimacy with their partner or a friend.

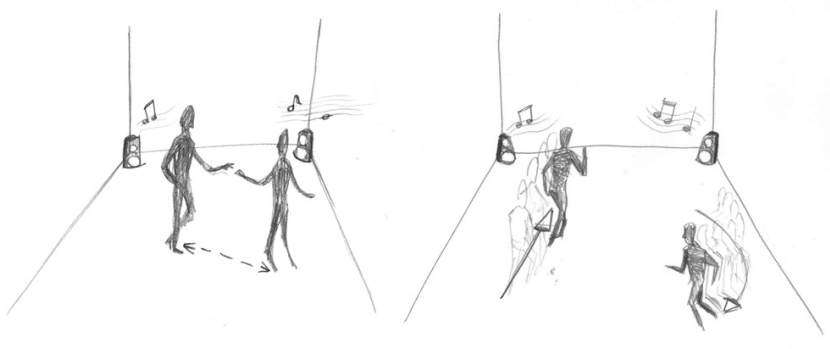

Figure 1. Sketched scenarios of The Music Room presented at the design workshop. In the left panel the pairs are dancing closer slowly. In the right panel they are running at a distance.

\subsection{Architecture}

The Music Room is composed of two main technological blocks: a tracking system and Robin, the algorithmic composer.

To track the position of visitors, we preferred a non-invasive solution based on computer vision approach [17 to wearable sensing solutions, as to avoid any physical constraint. The motion of the participants was monitored through a downwards-looking bird's-eye-view video camera installed on the ceiling of the room. The detection of the moving subjects was implemented by applying 
a standard background subtraction algorithm [17]. The obtained foreground information was then processed by the CamShift tracking algorithm [3]. The position of the participants returned by the tracking algorithm was progressively updated over time, and the extracted proxemic cues were supplied to the system for the purpose of providing information about the level of intimacy between them, which would in turn inform the music. Figure 2 displays a view of the room as seen by the camera.

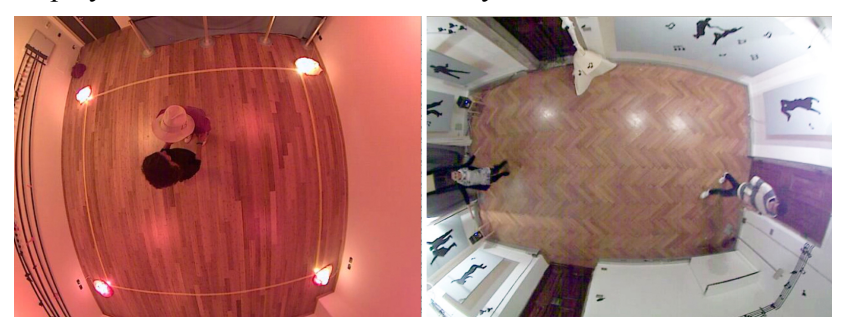

Figure 2. Two views of The Music Room from the camera mounted in the ceiling as recorded during two exhibitions.

Users can modulate distance and speed to communicate a different range of emotions. To do so, we adopted the circumflex model of emotions, which associates emotions with the the dimensions of valence (positive vs. negative) and arousal (activation vs. sleep) [28]. Following the metaphor of intimacy, low distance is mapped with positive emotions and high distance with negative emotions; high speed with intense emotion and low speed with mild emotions.

Following research in the psychology of music [16], the values of valence and arousal are then transformed into combinations of musical rules, which are used by Robin to adapt the musical flow in real time. Specifically, the emotional character of the music is continuously adjusted, modifying seven musical factors: mode, tempo, pitch contour, pitch register, theme recurrence, loudness, and consonance. In addition, for the purpose of increasing the variability and the quality of the composed music, different musical instruments were associated with different emotional conditions: the piano is constantly present in all conditions; a violin harmonises the piano voice when pairs are particularly close; a trombone harmonises the piano voice when pairs are on opposite sides of the room. Detailing the algorithmic composer is beyond the scope of this paper, but interested readers can find an in-depth description of Robin at [20].

Particular attention was paid to establishing the correct synchronisation between user movements and user reaction. Despite related work in interactive sonification suggests that latency should be as low as possible to keep the player engaged [14], we purposely decided to preserve the phraseological structure of music even in case of rapid changes in the emotional input. To this purpose, the successive musical phrase was computed at the last beat of the playing bar, which was fixed at $\frac{4}{4}$. This resulted in an approximately 4-second delay in the worst-case scenario, occurring when the current bar was at its first beat and playing at 60 BPM. Following the user feedback, we decided to reduce this latency, while still preserving musical coherence. To this end, at every quarter beat, a new input from the user was checked. If it ranked above or below a certain threshold, a new bar immediately started playing. This solution reduced the latency time to 1 second in the worst-case scenario.

${ }^{1}$ An extract of The Music Room, as viewed from the additional camera can be found at https://youtu.be/OSEvfjVivlw

\subsection{Field deployment}

The Music Room was first exhibited in Trento, Italy, at the 2012 edition of the Researchers' Night. This event involved 300 venues where academic and business research projects were publicly showcased. The event lasted from 5 PM to 2 AM, hosting almost 90 demonstrations and installations, which attracted a very heterogeneous audience. The Music Room was hosted by a $25 \mathrm{~m}^{2}$ classroom, which had been previously emptied of all furniture. Some minor adjustments were made to the room: to make the environment more pleasant, the walls were decorated with musical patterns. The room was originally illuminated by neon lights that did not seem to match the mood of the installation, which was intended to be more intimate and discreet. Therefore, we covered them with orange veils to both reduce the intensity of light and create a more enjoyable atmosphere. We wanted, indeed, to keep the room as dark as possible, as to foster intimacy. However, we had to accept a compromise solution, as the tracking system required a minimum level of lighting to function properly. Once the room was set up, we tested the tracking system. The test raised two issues: (i) the camera could not properly track the position of people when they were standing close to the walls; (ii) the neon lights were not powerful enough to correctly track the position of visitors. To tackle the first issue, the performance area was restricted and delimited with sticky tape. The second issue was addressed by adding four light bulbs, which were also covered with orange veils.

The second exhibition of The Music Room took place at the Museum of Science of Trento on the occasion of the 2013 edition of the ICT Days. The old, storied building located in the city centre provided an ideal setting for the installation. The $30 \mathrm{~m}^{2}$ room that hosted the installation was once again emptied of all furniture. A group of students and researchers volunteered to decorate the walls with musical patterns. The event lasted for 8 hours (from 2 PM to $6 \mathrm{PM}$ and from 9 PM to $1 \mathrm{AM})$. The building hosted several others exhibitions: in particular, late in the evening, the ground floor of the museum housed a disco music concert. The event, and particularly the concert, attracted a significant number of young people. Many of them visited the floor where The Music Room was also hosted and eventually tried the installation.

\section{EVALUATION}

Before entering the room, visitors were given detailed information about the functionality of the system: they could direct the music, which was generated by a computer, through their own movements. After this explanation, a researcher invited participants to sign a consent form stating that their session would be videotaped. Then, the researcher left the room and closed the door. Initially, people were free to experience the installation for as long as they wished. However, as in both events the number of visitors queuing to try the installation dramatically increased over time, at a certain point we had to limit the duration of each session.

Once people had left the room, two researchers asked them a few questions. On the occasion of the second exhibition, they were given the URL and the QR-code to access an online questionnaire, along with a personal code, to download the music they had created during the event. In addition to the camera installed on the ceiling of the room, which was used to track the participant movements, another camera was mounted in the room to videotape their performance from a different perspective ${ }^{1}$. This allowed the 
researchers to gain a better understanding of the behaviours and engagement of the participants.

The results presented are based on the integration of field observations, interviews, questionnaires, log-data and video analysis.

\subsection{Field Observations}

For both exhibitions, The Music Room was constantly busy for the whole duration of the event: 87 and 85 pairs participated, for a total of 344 visitors of all ages. Individual visits had an average duration of 5 minutes, ranging from a minimum of $1 \mathrm{~min} 30 \mathrm{~s}$ to a maximum of $10 \mathrm{~min}$ ). The installation met with a warm reception from visitors, who quickly started queuing up in front of the room. While putting off a few visitors, the long queue also caught the attention of several people, who eventually ended up joining the queue.

During the first exhibition, we were actually unprepared for such a success, but with the valuable help of six colleagues and friends we managed to handle the situation. As visitors started lining up to try the installation, they were invited to write their names on a waiting list. They were provided with an estimate of their waiting time, so that in the meantime they could continue their visit and come back to the room once their turn was actually approaching. Yet, at 2AM we had to open the room to the last curious visitors who were able to experience a quick and unpredictable group music creation.

Both nights were hectic and extremely intense. Fortunately, we experience hardly any technical incidents. The first exhibition taught us several important lessons about the logistics of our research settings. On the occasion of the second exhibition, in fact, we organised shifts involving a team of 13 researchers who worked hard throughout the whole event.

\subsection{Video Analysis}

A set of 30 videos displaying the behaviour of same-sex or opposite- sex pairs (10 for each of the three categories) was used to analyse engagement and collaboration. Four researchers independently watched the videos of each session and indicated an overall evaluation of engagement and collaboration on a 3-point scale. Collaboration ranged from 1-low (the participants pay little attention to each other) to 3-high (the participant make eye and voice contact, physically interact with each other and planning what to do next). Engagement ranged from 1-low (serious, bored) to 3high (smiling, dancing, jumping, running after each other, moving hands and arms, enthusiastic expressions). A weighted Cohen's Kappa was used to verify inter-rater variability. Kappa values for each pair of annotators ranged from fair (.3) to substantial (.62) for collaboration and from moderate (.5) to substantial (.78) for engagement. For each pair a measure of engagement and collaboration was computed by averaging individual evaluator scores.

Overall, $60 \%$ of the pairs proved highly collaborative. Around $30 \%$ of the pairs collaborated for most of the time, while $10 \%$ of them showed scarcely collaborative behaviours. The percentage related to the engagement level mirrored this scenario: $73 \%$ of the pairs looked engaged for the whole session, $10 \%$ reported average levels of engagement, while $17 \%$ did not express strong enthusiasm. We noticed gender to have an effect on collaboration: in particular, male pairs tended to collaborate less than male-female ones, and to occasionally focus on the technical infrastructure of the room. Some of them tried to tweak the system by black out the camera within their reach with their hands or clothes. Such attempt, however, never succeeded, as they were operating on the camera used for video recording only. No differences emerged on engagement level.

A second video analysis, based on a different set of videos $(\mathrm{N}=50)$ collected from both exhibitions equally, aimed at investigating the most recurring behaviours among visitors. Categories of behaviours were isolated and fine-tuned with the help of two researchers who independently viewed the video footages of each session several times. Results are summarised in Table 1, showing the percentage of pairs that performed one given behaviour and the percentage of time spent performing it. Alongside, the inter-rater agreement was computed using the Cohen's kappa. The kappa values range from 0.343 to 0.824 , with an average of 0.601 . Following the Altman interpretation of the kappa, the values then ranged from fair (0.21-0.40) to almost perfect (0.81-1.00), demonstrating that data were averagely reliable.

The four most common behaviours were walking, running, dancing, and standing still; all of these actions had a direct effect on the music played in the room. A considerable number of participants experimented with other playing behaviours, such as jumping, pirouetting, lying on the floor (and at times spinning or rolling), twisting, bowing, or mimicking a love declaration. These behaviours did not have a direct influence on the music.

Table 1: List of the most common behaviours with associated values of Kappa.

\begin{tabular}{|c|c|c|c|}
\hline Behaviours & $\%$ of time & $\%$ of pairs & kappa \\
\hline Walking & 36 & 96 & 0.613 \\
\hline Pair dancing & 17 & 76 & 0.762 \\
\hline Individual dancing & 11 & 56 & 0.690 \\
\hline Running & 10 & 82 & 0.612 \\
\hline Standing still & 8 & 100 & 0.522 \\
\hline Run and chase game & 6 & 64 & 0.641 \\
\hline Lie down & 3 & 7 & 0.824 \\
\hline Jumping & 2 & 47 & 0.599 \\
\hline Pirouetting & 2 & 32 & 0.612 \\
\hline Intimate behaviours & 2 & 32 & 0.409 \\
\hline Lift & 1 & 16 & 0.591 \\
\hline Fight & 0.5 & 28 & \multirow{5}{*}{0.343} \\
\hline Twisting & 0.5 & 28 & \\
\hline Bowing & 0.4 & 16 & \\
\hline Stamping the ground & 0.3 & 8 & \\
\hline Love declaration & 0.2 & 2 & \\
\hline
\end{tabular}

This analysis helped us to get a thorough insight into the visitor behaviours. Their engagement with the installation was to be clearly noticed in a number of performed actions; the high occurrence of several of these actions, however, indicated that they did not simply experience the installation as a music making activity. 
Table 2: Predicting behaviours by means of means and SD of distance and speed. In brackets, the percentage of pairs that reported that specific combination of distance and speed.

Low distance

Medium distance

High distance

\begin{tabular}{|c|c|c|c|}
\hline Low speed & $\begin{array}{c}\text { Intimate behaviours } \\
\text { Romantic dancing } \\
9.7 \%\end{array}$ & $\begin{array}{l}\text { Individual and collaborative } \\
\text { dancing } \\
12.9 \%\end{array}$ & $\begin{array}{c}\text { Individual behaviours } \\
9.7 \%\end{array}$ \\
\hline Medium speed & $\begin{array}{c}\text { Dancing } \\
\text { Walking together } \\
11.3 \%\end{array}$ & $\begin{array}{c}\text { Walking } \\
8.1 \%\end{array}$ & $\begin{array}{l}\text { Individual behaviours } \\
11.3 \%\end{array}$ \\
\hline High speed & $\begin{array}{l}\text { Pirouetting } \\
\text { Fighting }\end{array}$ & $\begin{array}{c}\text { Pursuit } \\
11.3 \%\end{array}$ & $\begin{array}{c}\text { Running } \\
14.3 \%\end{array}$ \\
\hline
\end{tabular}

$11.3 \%$

\subsection{Log Data Analysis}

A $\log$ data describing pairs' $(\mathrm{N}=63)$ position and speed was collected during the second exhibition and stored to be later analysed. The first analysis examined possible common performance patterns by analysing the movements of each dyad during the entire session: for each dyad, information on speed and distance was plotted and visually inspected with the help of two researchers. Visual inspection of the plots revealed that the interaction trajectories largely varied among pairs. Most pairs continuously changed their speed and distance, while in the minority of the cases proxemic cues varied to a very limited extent, suggesting that visitors continued to perform the same behaviours. Figure 3 shows the distance between the members of a dyad and their average speed as they varied during a session. This data was representative of a typical data set with respect to the continuous variability of the two dimensions.

A second analysis on the data focused on understanding whether the average values of distance and speed, collected during each session, could provide interesting insights into visitors' behaviours. For instance, intimate slow dancing could be represented by low means for both distance and speed, and running by high means for both factors. For the sake of convenience, the average values of distance and speed were divided into three categories with the $33^{\text {rd }}$ and $66^{\text {th }}$ percentiles. Table 2 maps combinations of the means for distance and speed with characteristic behaviours. Results revealed that the means for both distance and speed greatly varied among pairs. The nine combinations had a similar incidence (see Table 2), bearing witness to the great variety of behaviours manifested in the room. The most common combination was high speed with high distance (14\% of the pairs), associated with running or playing.

To better investigate this variability, the standard deviations (SDs) of distance and speed were analysed. Low SDs for both variables would suggest that visitors did not change their behaviours by any significant degree. Rather than exploring all interaction possibilities, they preferred to adopt a more passive behaviour. On the other hand, high SDs for both variables would suggest that visitors spent a significant percentage of their time experimenting with different combinations of speed and distance. The incidence of the two SDs showed that both variables exhibited a Gaussian distribution among the dataset of all the pairs, confirming that performed behaviours widely varied between and within pairs.

Log data were also analysed to evaluate potential differences among genre distribution. The means and SD values of distance and speed were entered as dependent variables into a MANOVA analysis with genre distribution (3: male-male, female-female, female, male) as the between-subjects factor. Results indicated that the dyad composition exerted a significant effect on average distance $(\mathrm{F}(2,60)=3.47, p<.1, \eta \mathrm{p} 2=.1)$ and speed standard deviation $(\mathrm{F}(2,60)=2.48, p<.1, \eta \mathrm{p} 2=.07)$. The effects were due to mixed-gender pairs, which interacted at a closer distance and varied their speed on a less frequent basis than male-male pairs. This result can be explained with reference to the gender stereotype of social acceptance of physical proximity in dancing situations.

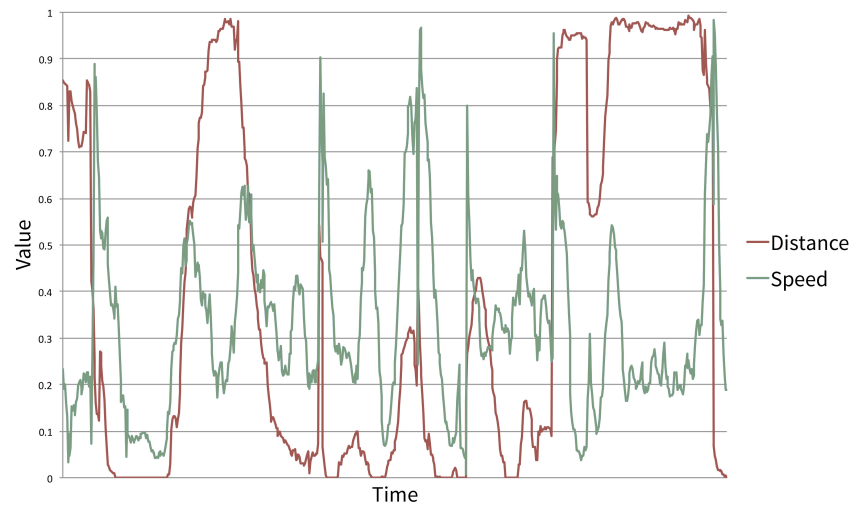

Figure 3: Speed and distance variability during a single session.

\subsection{Interviews}

At the first exhibition, 63 pairs were asked three questions and encouraged to express any further comments

The first question investigated how visitors had actually experienced the installation. Almost all visitors described it with flattering words (e.g. cool, interesting, unique, intimate, pleasant and relaxing). The only two visitors who did not enjoy the experience complained about a lack of interactivity: they were expecting a more direct manipulation of the artefact. Twelve pairs spontaneously reported that they were particularly impressed by the quality of the music.

The second question invited visitors to elaborate on the negative aspects of the experience, and suggest possible improvements. Once again, most people expressed flattering comments. The only concern, which was shared among 14 pairs, addressed a delay between their movements and the music reaction. On the occasion of the second exhibition, this issue had been improving the response of Robin to distance and speed changes (see Section 3.1).

The last question investigated the extent to which visitors perceived that they were in control of the music. This question highlighted an important dichotomy. Nearly half of the interviewees reported that 
they felt as if they were actively controlling the music. The other half declared that they were mainly following the music, only having the impression of playing an active role in a few situations. For instance, six pairs reported that they had initially spent some time controlling the music, but then had simply forgotten about the instructions and subsequently followed the music.

During the second exhibition, 77 pairs were interviewed. This time, we were mostly focused on understanding whether the ambivalence between controlling vs. following the music had been successfully tackled by the technical intervention on Robin. Results showed that this was partially the case, as $58 \%$ of the interviewees reported that they were controlling the music and another $27 \%$ experienced both feelings. Noticeably, only $15 \%$ felt like they were following the music.

\subsection{Questionnaires}

For the second exhibition, a total of 57 online questionnaires were collected from 32 female and 25 male respondents, 26\% of whom reported being capable of playing an instrument. The response rate was particularly high: $34 \%$ of all visitors responded to the questionnaire. The last page of the questionnaire featured a textbox where participants could enter the code they were given at the end of the session and download the song they had created in the room. They were also invited to visit our website to find information on our work, and to leave further comments on our Facebook page.

The seven questions were presented on a Likert scale ranging from 1 (I totally disagree) to 5 (I totally agree). The means and SDs are listed in Table 4. The music was generally appreciated (3.93), although some visitors would have preferred other musical genres. The most negative response regarded the number of available movements used to influence the music (2.77).

The data were analysed by means of a principal component factor analysis with Varimax rotation (Kaiser Normalisation). Two components with an eigenvalue greater than 1.0 were found. The components can be thought of as representing the general engagement with the experience (Component 1) and possible changes on the musical interaction (Component 2). The components loading are shown in Table 3.

A parametric test of correlation was then performed between selfreported musical expertise and the two components. There was a significant negative correlation between expertise and Component $1(\mathrm{r}=-.316, \mathrm{~N}=57, \mathrm{p}<.05)$, thus suggesting that untrained musicians had a more engaging experience. By contrast, the correlation between expertise and Component 2 was not significant, suggesting that all participants appreciated the music regardless of their music competence.

\subsection{System Quality and Reliability}

In addition to throwing light on the experience of the visitors, the field evaluation gave us feedback about the quality of the system. In particular, we investigated (i) how precisely the position of pairs was tracked, and (ii) how promptly Robin adapted its musical output in response to visitors' movements.

To obtain information about the accuracy of the tracking system, during both exhibitions, two researchers sat behind the control desk, observing the reaction of the system to the visitor. The visual tracking algorithm tracked their positions fairly accurately. Occasionally, when pair members were standing in close proximity for an extended period, the system was observed to lose track of one of them. In most cases, the system managed to fix this error very quickly.

With respect to Robin, interviews revealed that most visitors were impressed by the quality of the music, which was often described as barely distinguishable from that produced by a human musician. However, during the first exhibition, a brief latency between user movements and the generated music was reported. This latency was a consequence of a precise design choice: we intentionally decided to avoid sudden changes in music, as to preserve the phraseological structure of music. Still, in order to address this complaint, for the second edition of The Music Room, we modified the algorithm to reduce the latency, as reported in Section 3.1.

Table 3: The components found by the PCA, and variables that load on them with associated means and SDs.

\begin{tabular}{lcc} 
Component 1 & & mean (SD) \\
\hline "I enjoyed the installation" & .876 & $4.18(\mathrm{SD}=.98)$ \\
\hline $\begin{array}{l}\text { "I will recommend my friends } \\
\text { to trv this installation" }\end{array}$ & .801 & $4.33(\mathrm{SD}=1)$ \\
\hline $\begin{array}{l}\text { "It was a stimulating creative } \\
\text { experience" }\end{array}$ & .647 & $3.77(\mathrm{SD}=1.1)$ \\
\hline $\begin{array}{l}\text { "I did not like the music } \\
\text { inside the room" }\end{array}$ & .640 & $3.93(\mathrm{SD}=.98)$ \\
$\begin{array}{l}\text { "The music followed my and } \\
\text { my partner's movements" }\end{array}$ & .577 & $3.15(\mathrm{SD}=.97)$ \\
\hline \hline
\end{tabular}

\section{Component 2}

\begin{tabular}{lll}
\hline $\begin{array}{l}\text { "I would have preferred other } \\
\text { musical genres" }\end{array}$ & .876 & $3.02(\mathrm{SD}=1.51)$ \\
\hline $\begin{array}{l}\text { "The range of available } \\
\text { movements to influence music }\end{array}$ & .801 & $2.77(\mathrm{SD}=1.2)$
\end{tabular}
was too limited"

\subsection{Discussion}

Results collected from field observations, interviews, video analysis and questionnaires confirmed that a large percentage of visitors deeply enjoyed The Music Room. This enjoyment was due to a full range of different pleasurable behaviours, which were identified by $\log$ data and video analysis. The three scenarios originally envisioned in the design phase (i.e. music making, dancing and acting) occurred with different incidence. The most common scenario was dancing (performed by $76 \%$ of the pairs), probably because the synergy between music, movements and emotions is often associated with dancing. The acting scenario occurred only occasionally: during the interviews, only two pairs reported that they had been pretending to act in a theatre, and two other pairs mimicked love declarations, as shown by the video analysis. As opposed to these scenarios, the music making scenario was not easy to detect via video analysis, which failed to assess whether pairs ran, jumped, danced or walked to consciously influence the music. For instance, to make the music more tragic, dyad members could have simply walked away from each other or performed the same action while jumping. To create a serene musical output, instead, they could have simply stood in close proximity, dancing intimately. The interviews provided us with better insights into this topic. The question concerning the level of active involvement in the music process indeed precisely indicated the percentage of pairs who purposely tried to control the music.

The evaluation also revealed that, rather than simply focusing on making music, most visitors spent a notable amount of time performing actions that were not directly connected with music 
generation. Given the interactive dimensions at participants' disposal (i.e. distance and relative speed), the only gestures that would have a direct influence on the music were walking, running and standing. However, video analysis revealed that these gestures globally accounted for about half of the time. In fact, in addition to the original scenarios, the field studies disclosed a number of behaviours that had not been envisioned at the design stage. In particular, behaviours expressing delight and excitement occurred with a high incidence: pirouetting, twisting, and enactment of a fight were each performed by one out of three pairs. Furthermore, the analysis of the videos and the log data revealed that one pair out of three engaged in intimate behaviours such as romantic dancing, kissing, hugging and lifting, confirming the potential of The Music Room to facilitate intimate experiences.

These behaviours evidenced that visitors appropriated the installation in the most diverse ways, conceiving their own interpretation of it. This evidence is backed up by the study of [1], who described engagement in interactive art as a transformative dialogue between the participant and the artwork. This observation suggested that, in this design area, the actual status of the work could be defined and fully understood only when submitted to the verdict of an audience. In fact, The Music Room, ideated as an installation for music making, showed its status only when cross-checked against the interactions and the behaviours of users.

Finally, it is worth noting that while the techniques adopted in this study successfully accounted for the most recurring behaviours exhibited in the room, t they generally failed to explain the motivations behind them.

A cost-benefit analysis of the different data collection techniques is featured below:

- Field observations provided an initial understating of the engagement of the visitors. Following the framework proposed by [8] for understanding engagement with interactive art, this methodology contributed to identifying the attractors of the installation, i.e. the poster and videos placed all over the venue, the long queue and the smiling faces of people leaving the room.

- Video analysis proved crucial for assessing visitors' behaviours in the installations, allowing a precise understanding of the most common behaviours, as well as individual performances. However, performing accurate video analysis is a time-consuming method that entails engaging several researchers at a time. In addition, this information alone does not suffice to infer the driving motivations for visitors to engage in their selected activities.

- Log data analysis is a minimally time-consuming technique that might prove useful to gain a general knowledge of the variability of visitors' experiences with an interactive installation. In this case, it allowed us to acquire a better understanding of the variability of their experience, thus corroborating the thesis that The Music Room can foster diverse behaviours.

- Interviews helped to clarify visitors' behaviours. Collecting impromptu comments helped us to understand the first impressions of the visitors, i.e. the factors that most significantly caught their attention and sustained their engagement [8]. This approach, though, can be quite demanding to pursue on public exhibitions.

- Questionnaires: if properly administered, this is a lowbudget technique that can reveal interesting quantitative insights into visitors' experiences However, this methodology can only be effective if a sufficient number of entries are collected. Thus, a designer who is willing to exploit this technique should carefully ponder when and how to collect questionnaires. Administering questionnaires straight after the experience might help to maximise the number of filled-out questionnaires. Alternatively, online questionnaires might be administered taking the percentage of visitors that make the effort to go online at a later time to complete the questionnaires as an indicator of the' appreciation of this installation among the participants.

\section{CONCLUSION}

Only a handful of studies investigate $[13,19]$ investigate the evaluation of visitors' experience with interacting artworks. Indeed, the HCI community still needs to "provide meaningful answers as to what makes an engaging and meaningful art experience" [9]. Traditional HCI methods can effectively assess, among other aspects, the usability, the accessibility, and the userfriendliness of an interactive system. However, these might not be the right features to require of an artwork. Instead, researchers working in this domain should shift the focus of their investigations to other aspects. This paper takes an important step towards understanding these aspects, trying to find out what sorts of meanings people infer from The Music Room, how they understand it, and how they evaluate it as a creative activity, on their own terms.

This paper also proposes some key ideas that could be considered and applied to the development of interactive artworks, as well as strategies for evaluating and understanding the nature of such works. The systematic approach adopted for the evaluation of The Music Room expanded formal knowledge on principles of design in interactive art, which is much needed, as formal studies in this design field are scarce. In particular, a number of techniques for evaluating visitors experience with interactive art were reviewed, focusing on benefits and drawbacks. The result of this study proved that the formal evaluation of an interactive artwork could in fact provide numerous insights into the experience of the audience.

Interestingly, performing qualitative and quantitative analyses on user behaviours proved also helpful in framing the nature of the artwork. Our initial intention was to provide all users with an experience of music composition, but several people subverted it into an engaging, intimate and playful experience. This evidence encourages reflections on how to design for creative interaction and for appropriation. Our users were probably able to appropriate the installation because they were not required to perform any specific tasks: as the outcome of the interaction was not formally assessed, the experience was not biased by fear of failure in any way. This result suggests that designers and researchers working with interactive artworks should orchestrate visitors' interaction only to a limited extent, allowing room for improvisation and creative activities.

\section{ACKNOWLEDGMENTS}

This research was partially supported by the Inf@nzia Digi.tales 3.6 research grant by MIUR (Italian Ministry of Education, Universities and Research). We are much obliged to the members of the interaction research group for helping us to set up and run The Music Room. We are grateful to Valentina Caforio and Anna Berton for their help with the video analysis and to María Menéndez who contributed to the statistical analysis. Moreover, we wish to thank all the visitors who faced a long queue to experience The Music Room. Finally, we wish to acknowledge the contribution of Costanza Vettori, who edited the paper. 


\section{REFERENCES}

[1] Bilda, Z., Edmonds, E., \& Candy, L., 2008. Designing for creative engagement. Design Studies, 29(6), 525-540.

[2] Blaine, T., \& Fels, S. 2003. Collaborative musical experiences for novices.Journal of New Music Research, 32(4), 411-428.

[3] Bradski, G. R., 1998. Real time face and object tracking as a component of a perceptual user interface. In Applications of Computer Vision, 1998. WACV'98. Proceedings., Fourth IEEE Workshop on (pp. 214-219). IEEE.

[4] Camurri, A., Canepa, C., \& Volpe, G., 2007. Active listening to a virtual orchestra through an expressive gestural interface: The Orchestra Explorer. InProceedings of the 7th international conference on New interfaces for musical expression (pp. 56-61). ACM.

[5] Camurri, A., Varni, G., \& Volpe, G., 2010. Towards analysis of expressive gesture in groups of users: computational models of expressive social interaction. In Gesture in Embodied Communication and Human-Computer Interaction (pp. 122-133). Springer Berlin Heidelberg.

[6] Candy, L., Amitani, S., \& Bilda, Z., 2006. Practice-led strategies for interactive art research. CoDesign, 2(4), 209223.

[7] De Angeli, A., Sutcliffe, A., \& Hartmann, J. 2006. Interaction, usability and aesthetics: what influences users' preferences? In Proceedings of the 6th conference on Designing Interactive Systems. ACM.

[8] Edmonds, E., 2010. The art of interaction. Digital Creativity, 21(4), 257-264.

[9] England, D., 2012. Digital art and interaction: lessons in collaboration. In CHI'12 Extended Abstracts on Human Factors in Computing Systems (pp. 703-712). ACM.

[10] Fabiani, M., Dubus, G., \& Bresin, R., 2011. MoodifierLive: Interactive and collaborative expressive music performance on mobile devices. Proc. NIME 2011, 116-119.

[11] Fels, S., \& Mase, K. 1999. Iamascope: A graphical musical instrument. Computers \& Graphics, 23(2), 277-286.

[12] Hassenzahl, M. \& Tractinsky, N. 2006. User experience-a research agenda. Behaviour \& Information Technology 25.2 (2006): 91-97.

[13] Höök, K., Sengers, P., \& Andersson, G., 2003. Sense and sensibility: evaluation and interactive art. In Proceedings of the SIGCHI conference on Human factors in computing systems (pp. 241-248). ACM.

[14] Hunt, A., Hermann, T., \& Pauletto, S., 2004. Interacting with sonification systems: closing the loop. In Information Visualisation, 2004. IV 2004. Proceedings. Eighth International Conference on (pp. 879-884). IEEE.

[15] Jordà, S., Geiger, G., Alonso, M., \& Kaltenbrunner, M., 2007. The reacTable: exploring the synergy between live music performance and tabletop tangible interfaces. In Proceedings of the 1st international conference on Tangible and embedded interaction (pp. 139-146). ACM.

[16] Juslin, P. N., \& Sloboda, J. A., 2010. Music and Emotion: Theory, Research, Applications. Oxford University Press.
[17] KaewTraKulPong, P., \& Bowden, R., 2002. An improved adaptive background mixture model for real-time tracking with shadow detection. In Video-Based Surveillance Systems (pp. 135-144). Springer US.

[18] Kwastek, K. 2013. Aesthetics of interaction in digital art. MIT Press.

[19] Manovich, L. 2001. The language of new media. MIT press.

[20] Morreale, F., Masu, R., \& De Angeli, A. 2013. Robin: an algorithmic composer for interactive scenarios. Proceedings of SMC, 2013, 10th.

[21] Morreale, F., Masu, R., De Angeli, A., \& Rota, P. 2013. The music room. In CHI'13 Extended Abstracts on Human Factors in Computing Systems (pp. 3099-3102). ACM.

[22] Morreale, F., De Angeli, A., Masu, R., Rota, P., \& Conci, N. 2014. Collaborative creativity: The Music Room. Personal and Ubiquitous Computing, 18(5), 1187-1199.

[23] Morreale, F., De Angeli, A., \& O’Modhrain, S. 2014. Musical Interface Design: An Experience-oriented Framework. In Proc. of NIME (Vol. 14).

[24] Morrison, A. J., Mitchell, P., \& Brereton, M., 2007. The lens of ludic engagement: evaluating participation in interactive art installations. InProceedings of the 15th international conference on Multimedia (pp. 509-512). ACM.

[25] Patten, J., Recht, B., \& Ishii, H., 2002. Audiopad: a tag-based interface for musical performance. In Proceedings of the 2002 conference on New interfaces for musical expression (pp. 1-6). National University of Singapore.

[26] Polaine, A. 2005. The Flow Principle in Interactivity. Proceedings of the second Australasian conference on Interactive Environment. 151-58.

[27] Rowe, R. 1992. Interactive music systems: machine listening and composing. MIT press.

[28] Russell, J. A., 1980. A circumplex model of affect. Journal of personality and social psychology, 39(6), 1161.

[29] Sengers, P., \& Gaver, B., 2006. Staying open to interpretation: engaging multiple meanings in design and evaluation. In Proceedings of the 6th conference on Designing Interactive systems (pp. 99-108). ACM.

[30] van Boerdonk, K., Tieben, R., Klooster, S., \& van den Hoven, E., 2009. Contact through canvas: an entertaining encounter. Personal and Ubiquitous Computing, 13(8), 551567.

[31] Varni, G., Mancini, M., Volpe, G., \& Camurri, A., 2010. Sync'n'Move: social interaction based on music and gesture. In User Centric Media (pp. 31-38). Springer Berlin Heidelberg.

[32] Wanderley, M.M., \& N. Orio. 2002. "Evaluation of Input Devices for Musical Expression: Borrowing Tools from HCI." Computer Music Journal 26(3): 62-76.

[33] Zimmerman, J., Forlizzi, J., \& Evenson, S., 2007. Research through design as a method for interaction design research in HCI. In Proceedings of the SIGCHI conference on Human factors in computing systems (pp. 493-502). ACM. 